\title{
Impact of Oral Ubiquinol on Blood Oxidative Stress and Exercise Performance
}

\author{
Richard J. Bloomer, Robert E. Canale, Cameron G. McCarthy, and Tyler M. Farney
}

Cardiorespiratory-Metabolic Laboratory, The University of Memphis, Memphis, TN 38152, USA

Correspondence should be addressed to Richard J. Bloomer, rbloomer@memphis.edu

Received 5 April 2012; Accepted 4 June 2012

Academic Editor: Steve R. McAnulty

Copyright ( $) 2012$ Richard J. Bloomer et al. This is an open access article distributed under the Creative Commons Attribution License, which permits unrestricted use, distribution, and reproduction in any medium, provided the original work is properly cited.

Coenzyme Q10 (CoQ10) plays an important role in bioenergetic processes and has antioxidant activity. Fifteen exercise-trained individuals (10 men and 5 women; 30-65 years) received reduced CoQ10 (Kaneka QH ubiquinol; 300 mg per day) or a placebo for four weeks in a random order, double blind, cross-over design ( 3 week washout). After each four-week period, a graded exercise treadmill test and a repeated cycle sprint test were performed (separated by 48 hours). Blood samples were collected before and immediately following both exercise tests and analyzed for lactate, malondialdehyde, and hydrogen peroxide. Resting blood samples were analyzed for CoQ10 (ubiquinone and ubiquinol) profile before and after each treatment period. Treatment with CoQ10 resulted in a significant increase in total blood CoQ10 $(138 \% ; P=0.02)$ and reduced blood CoQ10 $(168 \% ; P=0.02)$, but did not improve exercise performance (with the exception of selected individuals) or impact oxidative stress. The relationship between the percentage change in total blood CoQ10 and the cycle sprint total work $\left(R^{2}=0.6009\right)$ was noted to be moderate to strong. We conclude that treatment with CoQ10 in healthy, exercise-trained subjects increases total and reduced blood CoQ10, but this increase does not translate into improved exercise performance or decreased oxidative stress.

\section{Background}

Coenzyme Q10 (CoQ10), referred to as ubiquinol in its most active $(\sim 95 \%)$ and reduced form [1], has documented roles related to bioenergetics and antioxidant activity [2]. Muscle function and subsequent physical performance during highstress conditions may be compromised due to a rise in reactive oxygen species (ROS) [3, 4], leading to an acute state of oxidative stress $[5,6]$. This is particularly true in middle to older age individuals who may experience increased oxidative stress [7], and may have some degree of CoQ10 deficiency [8], as compared to their younger counterparts.

Although the use of supplemental antioxidants to combat exercise-induced oxidative stress has been questioned recently when delivered as vitamin $\mathrm{E}$ and vitamin $\mathrm{C}[9$, 10], CoQ10 continues to be considered for this purpose. Specifically, it has been reported that supplementation with oral CoQ10 may minimize oxidative stress [11] and improve physical performance [12] in middle-aged adults. However, when investigating studies across a wide range of age groups, results have been mixed with some work documenting a performance benefit [13], and other work demonstrating little to no benefit following CoQ10 supplementation [14].

One possible explanation for the lack of evidence for CoQ10 to improve exercise performance may be the fact that traditional CoQ10 is poorly absorbed following oral ingestion [15]. This observation necessitates using high dosages of CoQ10 in order to obtain the desired physiological effects, something that has not been done in many of the published trials, with most daily dosages being less than or equal to $300 \mathrm{mg}$ [16]. One newly developed form of CoQ10 (Kaneka QH ubiquinol) has been reported to have excellent bioavailability [17], with a noted 4.7 -fold increase in plasma ubiquinol following single oral ingestion of CoQ10 at a dosage of $300 \mathrm{mg}$, and an approximate 10-fold increase following 28 days of treatment with CoQ10 at a daily dosage of $300 \mathrm{mg}$. Such an increase is far greater than what has been observed with traditional CoQ10 supplementation [18]. It is possible that such an improvement in bioavailability may translate into an improvement in physical performance, in 
addition to attenuation in potential exercise-induced oxidative stress.

The present study tested the influence of a bioavailable form of CoQ10, delivered as a dietary supplement in a sample of exercise-trained men and women aged 30-65 years, on plasma CoQ10 levels and markers of oxidative stress before and after 28 days of treatment. In addition, markers of exercise-induced oxidative stress were measured before and after single bouts of aerobic and anaerobic exercise, both preand postintervention, and measures of exercise performance were determined. We hypothesized that treatment with CoQ10 would increase plasma levels of CoQ10 and result in lower exercise-induced oxidative stress and improved exercise performance.

\section{Methods}

2.1. Subjects and Screening. Seventeen individuals initially enrolled in this study, but only 15 ( 10 men and 5 women) successfully completed all aspects of this work. Subjects completed health history, drug and dietary supplement usage, and physical activity questionnaires prior to enrollment. Subjects were physically active, regularly participating in aerobic and/or anaerobic exercise training. All subjects were instructed to maintain their prestudy exercise training program throughout the course of the intervention period. Subjects were nonsmokers, did not report any history of cardiovascular or metabolic disorders, and did not use nutritional supplements that might impact the outcome measures (or were willing to stop their use before and throughout the study period. One subject stopped using creatine two weeks prior to beginning the study). Subjects descriptive data are as follows: age: $42.7 \pm 10.4$ yrs; body mass: $75.2 \pm 16.3 \mathrm{~kg}$; years of anaerobic exercise training: $11.2 \pm 12.9$; hours per week of anaerobic exercise: $1.8 \pm 1.7$; years of aerobic exercise training: $14.0 \pm 13.7$; hours per week of aerobic exercise: $5.3 \pm 4.9$. Prior to participation, each subject was informed of all procedures, potential risks, and benefits associated with the study through both verbal and written form in accordance with the approved procedures of the University Institutional Review Board for Human Subjects Research (021011-357). Subjects provided verbal and written informed consent prior to being admitted into the study.

Subjects' body weight was measured, and heart rate and blood pressure were recorded following a 10-minute period of quiet rest (all values noted to be within normal limits). A maximal graded exercise test (GXT) was conducted using a treadmill and a sprint test was performed using an electronically braked cycle ergometer. These tests were performed as part of a familiarization procedure to prepare subjects for the exercise performance testing as described below. The tests were performed on different days, separated by 48 hours to allow for adequate recovery, and the procedures used were identical to those described below.

At the conclusion of the familiarization tests, a full explanation of dietary data recording was provided to subjects, along with data collection forms. An overview of all study procedures was also provided. Subjects were then assigned their initial condition (CoQ10 supplement or placebo), instructed on when to take the capsules, and scheduled for their remaining laboratory visits.

2.2. Supplementation. The study design involved a random order, double-blind, crossover assignment to a CoQ10 supplement (Kaneka QH ubiquinol; Kaneka Nutrients, Pasadena, TX; containing $300 \mathrm{mg}$ ubiquinol in a base of medium chain triglyceride oil, beeswax, soy lecithin, and ascorbyl palmitate) or placebo (medium chain triglyceride oil, beeswax, soy lecithin, and ascorbyl palmitate). Subjects ingested one capsule per day with breakfast, for a total of 30 days (28 days prior to the GXT and two-additional days leading up to the cycle sprint test-described below), with a 21-day wash out period between conditions. Prior work has indicated that a 28 -day treatment period with $300 \mathrm{mg} /$ day of supplemental Kaneka $\mathrm{QH}$ is more than adequate to elevate blood CoQ10 levels $(\sim 10$-fold above baseline), while a 14-day washout period is sufficient for returning blood CoQ10 levels to near presupplementation values [17]. Moreover, dosages up to $900 \mathrm{mg} /$ day for four weeks have been noted to be safe, without adverse events [19]. These findings are highlighted in the review by Hidaka and colleagues [20]. Both the supplement and placebo capsules were identical in appearance. For both conditions, capsules were distributed to subjects by research assistants in unlabeled bottles in amounts greater than needed for supplementation. Capsule counts upon bottle return allowed for estimation of compliance to intake.

2.3. Graded Exercise Test. Following each 28-day period of CoQ10 and placebo intake, subjects reported to the lab in the morning to perform a GXT on a treadmill, which allowed for the assessment of aerobic exercise performance. In an attempt to maintain consistency in testing, a script was read to each subject prior to performing the GXT. The protocol involved an increase in intensity every two minutes in the following manner: $\min 1-2,3.0 \mathrm{mph}, 0 \%$; $\min 3-4,3.5 \mathrm{mph}$, $0 \%$; $\min 5-6,4.0 \mathrm{mph}, 0 \%$; $\min 7-8,4.5 \mathrm{mph}, 0 \%$; $\min$ 9-10, $5.0 \mathrm{mph}, 0 \%$; $\min 11-12,5.0 \mathrm{mph}, 5 \%$; $\min 13-14$, $5.5 \mathrm{mph}$, 5\%; $\min 15-16,5.5 \mathrm{mph}, 7.5 \%$; min $17-18,6.0 \mathrm{mph}$, $7.5 \%$; $\min 19-20,6.0 \mathrm{mph}, 10 \%$; $\min 21-22,6.5 \mathrm{mph}, 10 \%$; $\min 23-24,6.5 \mathrm{mph}, 12.5 \%$; $\min 25-26,7.0 \mathrm{mph}, 12.5 \%$; $\min 27-28,7.0 \mathrm{mph}, 15 \%$; $\min 29-30,7.5 \mathrm{mph}, 15 \%$. No subject exceeded 29 minutes of exercise testing. This identical protocol was administered following each of the 4-week supplementation periods. During the final 15 seconds of each stage of exercise, heart rate was recorded via heart rate monitors and the Borg (6-20) scale of exertion was used to allow subjects to indicate their level of perceived work. Total exercise time was recorded. Although subjects performed the GXT in the morning following an overnight fast, they were allowed to drink water ad libitum before and following the GXT.

2.4. Cycle Sprint Test. On a separate day from the GXT (within 48 hours), subjects returned to the lab in the morning to perform 5-repeated cycle sprints on an electronically 
braked cycle ergometer (Lode Excaliber Sport; Groningen) using a fixed resistance based on body mass (torque factor: $0.7 \mathrm{Nm} / \mathrm{kg}$ ). This test was allowed for the assessment of anaerobic exercise performance. Each sprint was 10 seconds in duration and two minutes of active recovery was allowed between each sprint. For each sprint, total work $(\mathrm{kJ})$ and power output were calculated. Heart rate and perceived exertion were also measured following each sprint. While subjects performed the cycle sprint test in the morning following an overnight fast, they were allowed to drink water ad libitum before and following the test. As with the GXT, a script was read to each subject prior to performing the sprint test and the exact procedures for testing were used after each four-week period of supplementation. After the performance of the GXT and on the day between the maximal exercise tests, subjects continued with their assigned condition (CoQ10 or placebo), which provided a total of 30 days of treatment.

2.5. Blood Sampling. Venous blood samples $(\sim 20 \mathrm{~mL})$ were collected from subjects' forearm via needle and Vacutainer before and following each period of supplementation with CoQ10 and placebo. Samples were collected at rest in a 10hour fasted state, following a 10-minute period of quiet rest. Preintervention blood samples were analyzed for measures of oxidative stress, in addition to a CoQ10 profile. Following each intervention period, blood was collected before and immediately after each exercise test and analyzed for whole blood lactate, plasma malondialdehyde, and plasma hydrogen peroxide. Samples collected in tubes with EDTA were used for the analysis of whole blood lactate (Lactate Plus; Nova Biomedical, Waltham, MA) and the remaining blood was immediately centrifuged at $1500 \mathrm{~g}$ at $4^{\circ} \mathrm{C}$ to obtain plasma. Samples for analysis of CoQ10 profile were collected in sodium heparinized tubes and immediately centrifuged at $1500 \mathrm{~g}$ at $4^{\circ} \mathrm{C}$ to obtain plasma. Following centrifugation, the plasma was immediately stored in multiple aliquots at $-70^{\circ} \mathrm{C}$.

2.6. Biochemistry. Malondialdehyde was analyzed in plasma using a commercially available colorimetric assay (Northwest Life Science Specialties; Vancouver, WA), using a modified method described by Jentzsch et al. [21], which is suggested to improve the sensitivity of this assay. Despite the improved sensitivity, other lipid specific markers such as 8-isoprostanes and hexanoyl-lysine could have been considered in this work, as these assays have been suggested as more sensitive markers of lipid peroxidation as compared to malondialdehyde. Our failure to include these other markers could be considered a limitation of this work. For analysis of malondialdehyde, plasma samples were added to microcentrifuge reaction tubes with the addition of $1 \mathrm{M}$ phosphoric acid and 2thiobarbituric acid reagent. Samples and reagents were incubated for 60 minutes at $60^{\circ} \mathrm{C}$. Following incubation and removal of the reaction tubes, the mixture was transferred to a microplate, and the absorbance was read using a spectrophotometer at both 535 and $572 \mathrm{~nm}$. Quantification was performed with a calibration curve using tetramethoxypropane in a stabilizing buffer. Hydrogen peroxide was analyzed in plasma using the Amplex Red reagent method as described by the manufacturer (Molecular Probes; Invitrogen Detection Technologies, Eugene, OR). In the reaction mixture, hydrogen peroxide, in the presence of horseradish peroxidase, reacts with Amplex Red reagent to generate the red-fluorescence oxidation product, resorufin. Quantification was performed with a hydrogen peroxide calibration curve. Oxidative stress biomarker samples were analyzed in duplicate on first thaw. CoQ10 profile was analyzed in plasma using high-performance liquid chromatography in the Neuropharmacology and Clinical Laboratories of Cincinnati Children's Hospital Medical Center, using the procedures previously described in detail by Tang and colleagues [22].

2.7. Perceived Vigor. Subjects were asked to rate their perceived vigor during the past three weeks using a visual scale (where $10=$ very energetic and $0=$ very nonenergetic) at weeks $0,4,7$, and 11 . These times coincided with pre- and postintervention for both supplement and placebo.

2.8. Dietary Intake and Physical Activity. All subjects were instructed to maintain their normal diet, without attempts to increase or decrease antioxidant nutrient intake. Subjects completed a four-day food log surrounding the days of testing ( 2 days before the GXT, the day of the GXT, and the day after the GXT). All calorie containing foods and drinks were recorded. Nutritional records were analyzed for total calories, protein, carbohydrate, fat, and a variety of micronutrients (Food Processor SQL, version 9.9, ESHA Research, Salem, OR). Subjects were given specific instructions regarding abstinence from alcohol consumption during the 48 hours immediately prior to the test days. They were also instructed to maintain their normal physical activity, with the exception of refraining from strenuous physical activity during the 48 hours preceding each test day.

2.9. Statistical Analysis. For the analysis of resting blood measures and perceived vigor, outcome variables were analyzed using a 2 (condition) $\times 2$ (pre-/postintervention) analysis of variance (ANOVA). For exercise performance data, variables were analyzed using a one-way ANOVA (for condition). For exercise bloodborne variables, data were analyzed using a 2 (condition) $\times 2$ (time-pre-/postexercise) ANOVA. Due to our unequal and small sample sizes for men and women, no formal attempt was made to compare the response between sexes; however, this was done in a cursory manner for the two-exercise-performance measures (GXT time and cycle total work). Tukey post hoc tests were used as needed. Dietary data were analyzed using a one-way ANOVA (for condition). All analyses were performed using JMP statistical software (version 4.0.3, SAS Institute, Cary, NC). Statistical significance was set at $P \leq$ 0.05 . The data are presented as mean \pm SEM, except for subject descriptive characteristics (mean $\pm \mathrm{SD}$ ). 
TABLE 1: Dietary intake of 15 exercise-trained men and women during the days surrounding treadmill and cycle testing following treatment with CoQ10 or placebo.

\begin{tabular}{lccc}
\hline Variable & CoQ10 & Placebo & $P$ value \\
\hline Kilocalories & $2083 \pm 188$ & $2131 \pm 181$ & 0.85 \\
Protein (g) & $87 \pm 8$ & $86 \pm 7$ & 0.91 \\
Protein (\%) & $17 \pm 1$ & $17 \pm 1$ & 0.63 \\
Carbohydrate (g) & $256 \pm 25$ & $255 \pm 24$ & 0.97 \\
Carbohydrate (\%) & $48 \pm 3$ & $48 \pm 3$ & 0.95 \\
Fiber (g) & $18 \pm 3$ & $19 \pm 2$ & 0.86 \\
Sugar (g) & $92 \pm 15$ & $95 \pm 14$ & 0.89 \\
Fat (g) & $79 \pm 10$ & $84 \pm 10$ & 0.72 \\
Fat (\%) & $34 \pm 2$ & $34 \pm 2$ & 0.90 \\
Saturated Fat (g) & $26 \pm 4$ & $28 \pm 4$ & 0.68 \\
Monounsaturated fat (g) & $15 \pm 3$ & $13 \pm 3$ & 0.45 \\
Polyunsaturated fat (g) & $7 \pm 1$ & $5 \pm 1$ & 0.19 \\
Cholesterol (mg) & $258 \pm 45$ & $287 \pm 43$ & 0.65 \\
Vitamin C (mg) & $56 \pm 9$ & $53 \pm 8$ & 0.80 \\
Vitamin E (mg) & $4 \pm 0$ & $3 \pm 0$ & 0.09 \\
Vitamin A (RE) & $385 \pm 87$ & $306 \pm 83$ & 0.52 \\
\hline
\end{tabular}

Values are mean \pm SEM.

\section{Results}

3.1. Compliance, Vigor, and Dietary Data. Of the initial 17-enrolled subjects, one man and one woman failed to complete all aspects of the study due to personal reasons with scheduling. The remaining 15 subjects successfully completed testing, with the exception of one woman not completing the GXT with both conditions, and one woman not completing the cycle sprint test with placebo. Regarding compliance to capsule intake, subjects were $93 \pm 4 \%$ compliant to CoQ10 capsules and $98 \pm 1 \%$ compliant to placebo capsules, with no statistical difference noted between conditions $(P=0.20)$. Regarding perceived vigor, no condition $(P=0.24)$, pre-/postintervention $(P=0.92)$, or interaction $(P=0.79)$ effect was noted, with values for CoQ10 (pre: $7.4 \pm 0.5$ and post: $7.3 \pm 0.5$ ) and placebo (pre: $6.8 \pm 0.4$ and post: $6.8 \pm 0.5$ ) near identical from pre- to postintervention. Regarding dietary intake, no difference was noted between conditions for any presented variable $(P>$ 0.05). Dietary data are displayed in Table 1.

3.2. Exercise Test Data. No differences were noted between CoQ10 and placebo for exercise performance and related variables (heart rate and perceived exertion), with near identical mean values observed for all variables $(P>0.05)$. However, selected subjects did experience an improvement in GXT exercise time and cycle sprint test total work, as shown in Figure 1. Subjects appeared to work very hard during testing, as evidenced by a mean peak heart rate on the GXT of 180 beats per minute (above the age-predicted maximum) and a rating of perceived exertion value of close to 20/20. Total GXT time was over 22 minutes, which is considered excellent given the protocol used and the age of the subjects, and was not different $(P=0.51)$ for men $(22.3$ minutes $)$ and women (21.0 minutes). Total work during the sprint cycle test was approximately $5000 \mathrm{~kJ}$, and was greater $(P<0.0001)$ for men $(5589 \mathrm{~kJ})$ as compared to women $(3514 \mathrm{~kJ})$, which was expected based on the larger body mass of men. Despite these findings for GXT exercise time and total work during the sprint cycle test, no sex $\times$ condition interactions were noted for the GXT $(P=0.88)$ or sprint cycle test $(P=0.72)$. Data for the GXT are presented in Table 2(a), while data for the cycle sprint test are presented in Table 2(b). Individual subject data for total treadmill time and total work for the sprint cycle test are presented in Figure 1.

3.3. Resting Oxidative Stress and CoQ10 Profile. No differences were noted between CoQ10 supplementation and placebo for measures of oxidative stress at rest, before and after the 4 -week intervention period $(P>0.05)$. However, treatment with CoQ10 resulted in a significant increase in total blood CoQ10 (138\%; $P=0.02)$ and reduced blood CoQ10 (168\%; $P=0.02$ ) from pre- to postsupplementation. Due to the increase in total CoQ10 and the stable cholesterol values from pre- to postintervention, the CoQ10: Cholesterol ratio was increased significantly $(P=$ $0.01)$. Values for these parameters with placebo treatment remained relatively constant, with a slight decrease noted. No other variables within the CoQ10 profile were altered significantly $(P>0.05)$. Data are presented in Table 3 . Although not a main focus of the present research, the relationship between the percentage change in total blood CoQ10 and exercise performance (placebo versus CoQ10 supplement) was noted for GXT time $\left(R^{2}=0.0744 ; P=\right.$ $0.35)$ and cycle sprint total work $\left(R^{2}=0.6009 ; P=0.001\right)$. Data are presented in Figure 2.

3.4. Exercise-Induced Oxidative Stress and Lactate. No differences were noted between CoQ10 and placebo for measures of oxidative stress in response to exercise $(P>0.05)$, with only a moderate oxidative stress observed in response to both the GXT and the cycle sprint test. With the GXT, the time effect approached significance for malondialdehyde $(P=$ $0.11)$ and hydrogen peroxide $(P=0.06)$, while the same was true for malondialdehyde $(P=0.09)$ but not hydrogen peroxide $(P=0.47)$ with regard to the cycle sprint test. No condition or condition $\times$ time effects were noted for blood lactate in regard to either exercise test $(P>0.05)$. However, a time effect was noted for blood lactate for both exercise tests $(P<0.0001)$, with a significant increase from pre- to postexercise. Data are presented in Table 4.

\section{Discussion}

Findings from the present investigation indicate that supplementation of CoQ10 in healthy, exercise-trained men and women increases total and reduced blood CoQ10, in addition to CoQ10: Cholesterol ratio. Neither an improvement in exercise performance is noted, nor does supplementation result in attenuation of resting or exercise-induced oxidative stress biomarkers. These data are specific to a sample of 
TABle 2: Heart rate, perceived exertion, and performance data of exercise-trained men and women during treadmill testing (a) and cycle sprints (b) following treatment with CoQ10 or placebo.

(a)

\begin{tabular}{lccc}
\hline Variable* & CoQ10 & Placebo & $P$ value \\
\hline Heart rate min 8 (bpm) & $119.9 \pm 6.4$ & $122.2 \pm 5.7$ & 0.79 \\
Heart rate min 14 (bpm) & $148.2 \pm 6.5$ & $147.1 \pm 5.0$ & 0.87 \\
Heart rate peak (bpm) & $180.1 \pm 3.5$ & $180.5 \pm 3.1$ & 0.98 \\
Perceived exertion min 8 (6-20 scale) & $11.4 \pm 0.4$ & $11.9 \pm 0.3$ & 0.50 \\
Perceived exertion min 14 (6-20 scale) & $13.9 \pm 0.6$ & $14.3 \pm 0.6$ & 0.91 \\
Perceived exertion peak (6-20 scale) & $19.5 \pm 0.2$ & $19.7 \pm 0.1$ & 0.37 \\
Total treadmill time (sec) & $1324.5 \pm 69.3$ & $1317.3 \pm 62.0$ & 0.94 \\
\hline
\end{tabular}

(b)

\begin{tabular}{lccc}
\hline Variable** & CoQ10 & Placebo & $P$ value \\
\hline Heart rate (bpm) & $155.6 \pm 3.6$ & $156.4 \pm 3.2$ & 0.96 \\
Perceived exertion (6-20 scale) & $17.6 \pm 0.3$ & $16.9 \pm 0.4$ & 0.19 \\
Peak power (watts) & $875.0 \pm 63.3$ & $914.7 \pm 57.2$ & 0.65 \\
Mean power (watts) & $483.3 \pm 35.2$ & $511.0 \pm 36.9$ & 0.59 \\
Total work (kJ) & $4833.1 \pm 351.6$ & $5109.8 \pm 368.9$ & 0.59 \\
\hline
\end{tabular}

Values are mean \pm SEM.

*Heart rate and perceived exertion values taken during the final 15 seconds of minute 8 (stage 4) and minute 14 (stage 7) of treadmill testing; peak values for heart rate and perceived exertion taken at the end of the treadmill testing.** Values are averages taken over five-, 10-second cycle sprints.

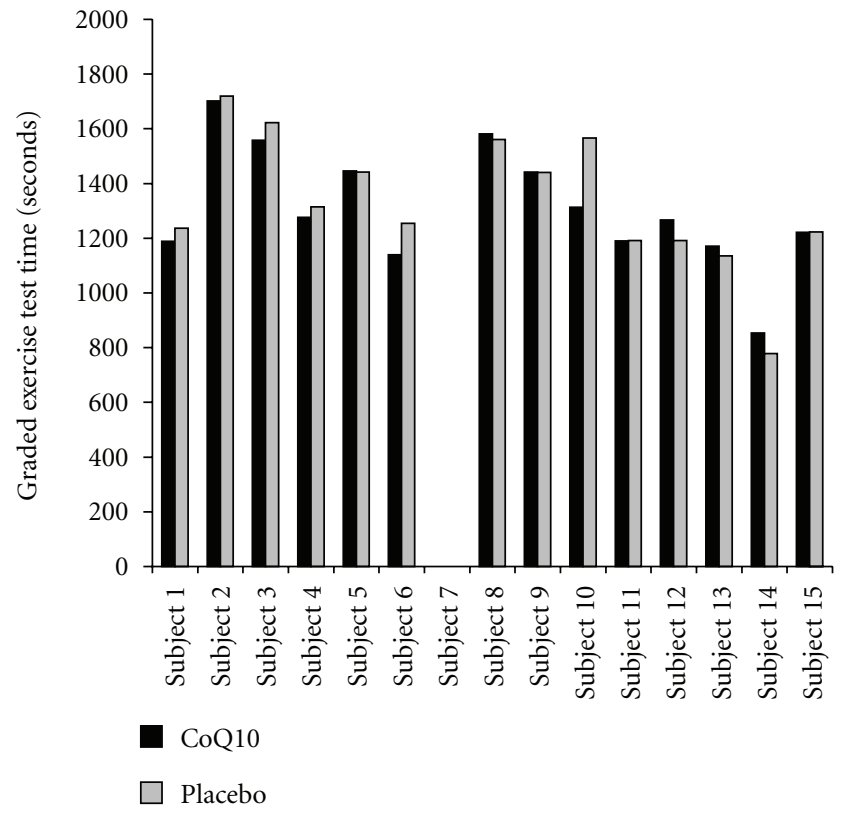

(a)

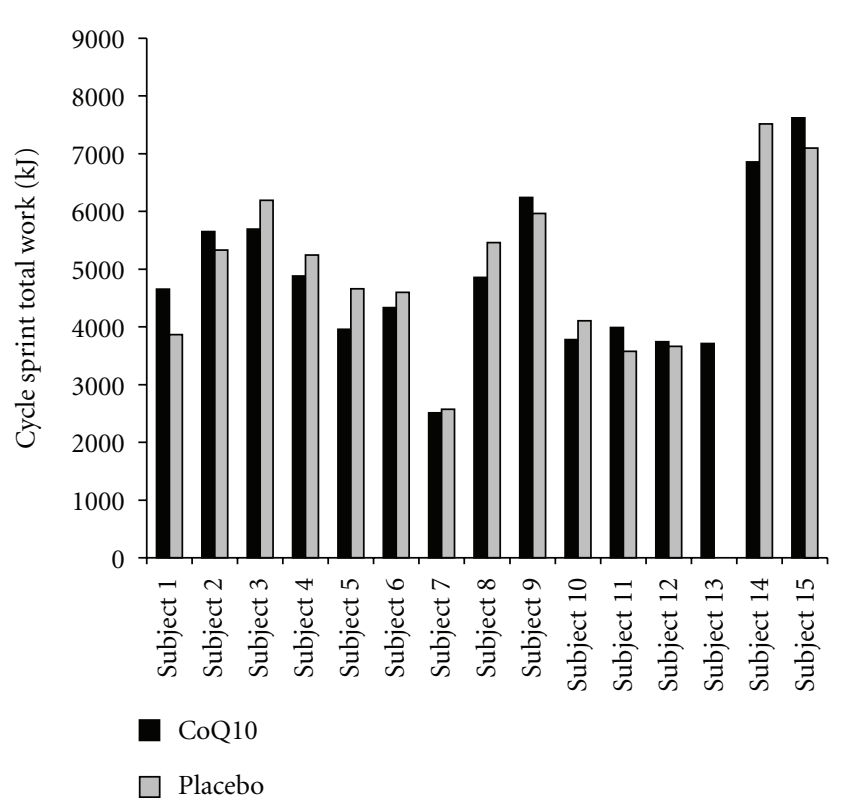

(b)

FIgURE 1: Treadmill time (a) and total work during cycle testing (b) of exercise-trained men and women following treatment with CoQ10 or placebo. Note: Subjects 7, 10,11, 12, and 13 are women.

healthy, exercise-trained men and women supplementing CoQ10 at a daily dosage of $300 \mathrm{mg}$.

4.1. Plasma CoQ10. Treatment with CoQ10 resulted in a significant increase in plasma CoQ10 concentrations, which supports the prior work of Hosoe et al. [17], who found that the same dosage and time of administration increases plasma ubiquinol approximately 10-fold. The baseline plasma ubiquinol values in that study involving healthy subjects was 0.57 to $0.66 \mu \mathrm{g} \cdot \mathrm{mL}^{-1}$, lower than what we observed in the present study. Although we did not observe such a magnitude of increase, our subjects did indeed experience a significant rise in total and reduced CoQ10 (Table 3 ). It is possible that the variance could be attributed 
TABle 3: Oxidative stress and Coenzyme Q10 blood profile (a) and statistical findings (b) of exercise-trained men and women before (Pre) and after (Post) treatment with CoQ10 or placebo.

(a)

\begin{tabular}{|c|c|c|c|c|}
\hline \multirow{2}{*}{ Variable } & CoQ10 & CoQ10 & Placebo & Placebo \\
\hline & Before & After & Before & After \\
\hline Malondialdehyde $\left(\mu \mathrm{mol} \cdot \mathrm{L}^{-1}\right)$ & $0.87 \pm 0.12$ & $0.89 \pm 0.11$ & $0.92 \pm 0.12$ & $0.89 \pm 0.12$ \\
\hline Hydrogen Peroxide $\left(\mu \mathrm{mol} \cdot \mathrm{L}^{-1}\right)$ & $2.72 \pm 0.34$ & $3.23 \pm 0.32$ & $2.92 \pm 0.35$ & $3.36 \pm 0.34$ \\
\hline Total CoQ10 $\left(\mu \mathrm{g} \cdot \mathrm{mL}^{-1}\right)$ & $0.98 \pm 0.10$ & $2.33 \pm 0.42$ & $1.02 \pm 0.11$ & $0.99 \pm 0.09$ \\
\hline Reduced CoQ10 $\left(\mu \mathrm{g} \cdot \mathrm{mL}^{-1}\right)$ & $0.82 \pm 0.08$ & $2.13 \pm 0.39$ & $0.87 \pm 0.09$ & $0.84 \pm 0.07$ \\
\hline Reduced CoQ10 (\%) & $84.41 \pm 2.10$ & $90.62 \pm 1.63$ & $85.64 \pm 2.38$ & $85.96 \pm 1.93$ \\
\hline Oxidized CoQ10 $\left(\mu \mathrm{g} \cdot \mathrm{mL}^{-1}\right)$ & $0.17 \pm 0.03$ & $0.21 \pm 0.04$ & $0.15 \pm 0.04$ & $0.15 \pm 0.03$ \\
\hline Total Cholesterol $\left(\mathrm{mg} \cdot \mathrm{dL}^{-1}\right)$ & $199.33 \pm 9.32$ & $197.07 \pm 9.55$ & $198.80 \pm 10.34$ & $200.27 \pm 12.25$ \\
\hline CoQ10: Cholesterol & $0.48 \pm 0.39$ & $1.13 \pm 0.15$ & $0.51 \pm 0.04$ & $0.49 \pm 0.04$ \\
\hline
\end{tabular}

Values are mean \pm SEM.

(b)

\begin{tabular}{lccc}
\hline Variable & $\begin{array}{c}\text { Condition } \\
P \text { value }\end{array}$ & $\begin{array}{c}\text { Before/after } \\
P \text { value }\end{array}$ & $\begin{array}{c}\text { Condition } \times \text { Before/after } \\
P \text { value }\end{array}$ \\
\hline Malondialdehyde & 0.79 & 0.92 & 0.83 \\
Hydrogen Peroxide & 0.62 & 0.17 & 0.92 \\
Total CoQ10 & $0.02^{*}$ & $0.04^{*}$ & 0.13 \\
Reduced CoQ10 & $0.02^{*}$ & $0.03^{*}$ & 0.11 \\
Reduced CoQ10\% & 0.30 & 0.10 & 0.42 \\
Oxidized CoQ10 & 0.50 & 0.97 & 0.47 \\
Total Cholesterol & 0.62 & 0.55 & 0.96 \\
CoQ10: Cholesterol & $0.01^{*}$ & $0.01^{*}$ & $0.00^{*}$ \\
\hline
\end{tabular}

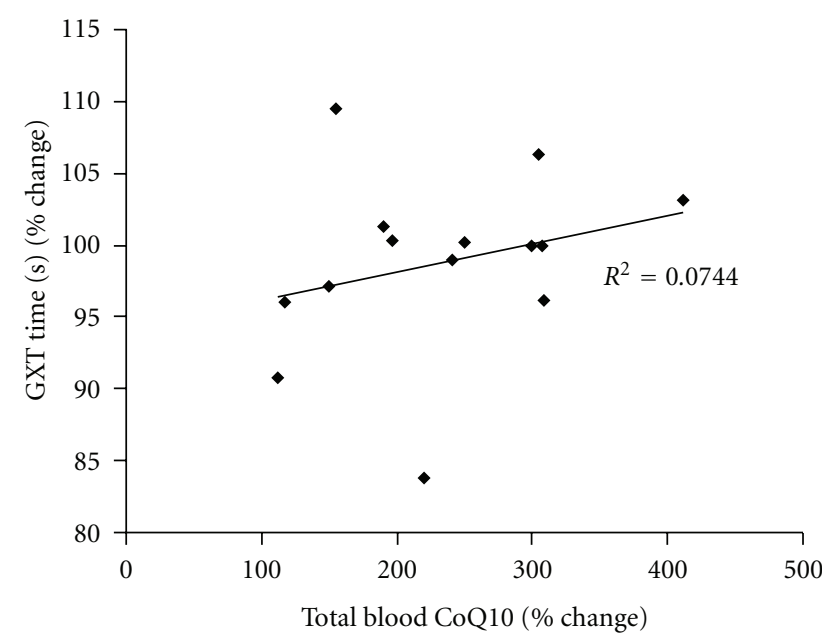

(a)

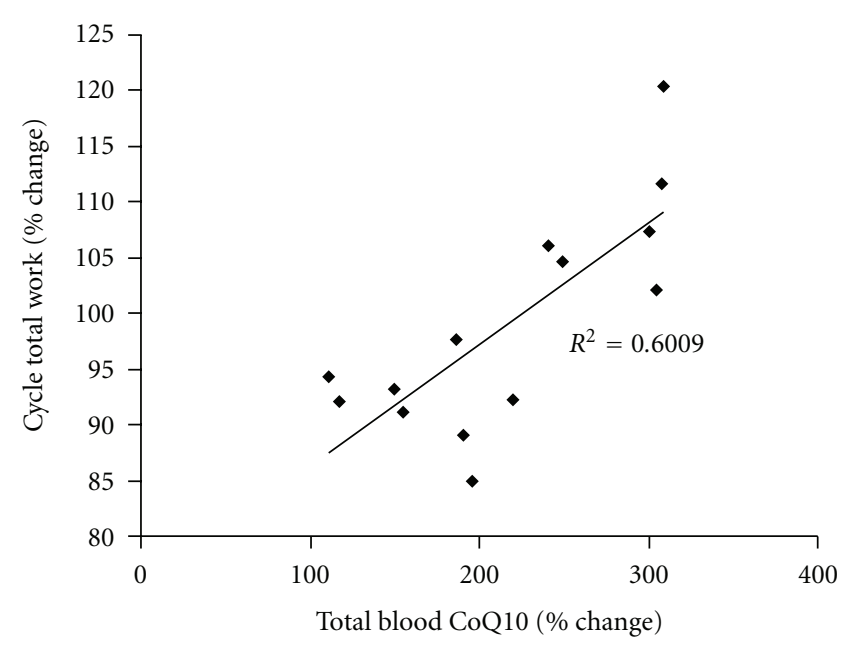

(b)

FIgURE 2: Relationship between the percentage change in GXT time (a) and cycle total work (b) and total blood CoQ10. Note: The percentage change value was calculated between placebo versus CoQ10.

to a difference in formulation, and that a greater magnitude of increase in plasma CoQ10 could result in more favorable findings for our other outcome measures, as described below. Based on the role of CoQ10 in regulating metabolic function and the impact of cholesterol on vascular health, our finding of an increase in CoQ10: Cholesterol ratio (i.e., normalization of CoQ10 for cholesterol concentrations) may have implications for improved cardiovascular and metabolic health, as indicated previously for conditions such as advanced congestive heart failure.

4.2. Resting Oxidative Stress. CoQ10 supplementation has been reported to lower biomarkers of oxidative stress when 
TABLE 4: Oxidative stress and blood lactate of exercise-trained men and women before (pre) and after (post) treadmill and cycle exercise, following treatment with CoQ10 or placebo.

\begin{tabular}{lccc}
\hline Time & Malondialdehyde $\left(\mu \mathrm{mol} \cdot \mathrm{L}^{-1}\right)$ & Hydrogen Peroxide $\left(\mu \mathrm{mol} \cdot \mathrm{L}^{-1}\right)$ & Lactate* $\left.^{*} \mathrm{mmol} \cdot \mathrm{L}^{-1}\right)$ \\
\hline Treadmill (before) CoQ10 & $0.89 \pm 0.11$ & $3.23 \pm 0.32$ & $1.25 \pm 0.56$ \\
Treadmill (after) CoQ10 & $0.93 \pm 0.11$ & $4.61 \pm 0.53$ & $8.83 \pm 0.58$ \\
Treadmill (before) Placebo & $0.89 \pm 0.12$ & $3.36 \pm 0.34$ & $1.33 \pm 0.56$ \\
Treadmill (after) Placebo & $1.05 \pm 0.10$ & $4.14 \pm 0.49$ & $8.36 \pm 0.53$ \\
Cycle (before) CoQ10 & $0.81 \pm 0.10$ & $3.54 \pm 0.53$ & $1.12 \pm 0.53$ \\
Cycle (after) CoQ10 & $0.98 \pm 0.10$ & $3.86 \pm 0.52$ & $10.36 \pm 0.51$ \\
Cycle (before) Placebo & $0.92 \pm 0.10$ & $3.58 \pm 0.59$ & $1.18 \pm 0.57$ \\
Cycle (after) Placebo & $1.08 \pm 0.10$ & $4.10 \pm 0.59$ & $10.06 \pm 0.57$ \\
\hline
\end{tabular}

Values are mean \pm SEM.

Treadmill:

No condition $(P=0.95)$, time $(P=0.11)$, or condition $\times$ time $(P=0.23)$ effect for MDA.

No condition $(P=0.41)$, time $(P=0.06)$, or condition $\times$ time $(P=0.94)$ effect for $\mathrm{H}_{2} \mathrm{O}_{2}$.

No condition $(P=0.75)$ or condition $\times$ time $(P=0.61)$ effect for lactate; ${ }^{*}$ Time effect $(P<0.0001)$ for lactate.

Cycle:

No condition $(P=0.30)$, time $(P=0.09)$, or condition $\times$ time $(P=0.94)$ effect for MDA.

No condition $(P=0.82)$, time $(P=0.47)$, or condition $\times$ time $(P=0.87)$ effect for $\mathrm{H}_{2} \mathrm{O}_{2}$.

No condition $(P=0.84)$ or condition $\times$ time $(P=0.73)$ effect for lactate; Time effect $(P<0.0001)$ for lactate.

measured at rest [11,23]. For example, Niklowitz et al. investigated the changes in 8-hydroxydeoxy-guanosine (8-OHdG) after 28 days of CoQ10 supplementation at $3 \mathrm{mg} / \mathrm{kg} /$ day [11]. The authors reported a delayed formation of 8-OHdG over the course of study. Also investigating the role of CoQ10 on oxidative stress biomarkers, Weber and colleagues supplemented healthy subjects with $90 \mathrm{mg} /$ day of CoQ10 and reported a decrease in TBARS after two weeks of treatment [23]. In the present study, it is possible that our lack of a difference between pre- and postsupplementation of CoQ10 for oxidative stress biomarkers was due to the fact that all measures were relatively low to begin with, as we have recently reported for active men and women [24]. Despite any potential antioxidant effect of the CoQ10, there may have been little need to further decrease the already low-resting levels of these oxidative stress biomarkers. This is particularly true when considering that a mild degree of ROS generation (and oxidative stress) appears a vital component of normal physiological functioning [25]. Additionally, the baseline total CoQ10 levels of our subjects were relatively high and could have impacted the benefit of the supplementation. Perhaps the inclusion of diseased and/or older subjects would have allowed us to detect a significant effect of CoQ10 treatment.

4.3. Exercise-Induced Oxidative Stress. Aside from resting conditions, several investigations have been conducted focused on attenuating exercise-induced oxidative stress via use of antioxidant treatment. Pertaining to this line of research, results from these studies are largely mixed $[6,26$, 27], and are likely dependent on the type, dosage, and time frame of treatment of the antioxidant(s), the tissue sampled, the exercise protocol used to induce oxidative stress, the time of measurement, the assays used, and the test subjects recruited (i.e., trained versus untrained, old versus young, and healthy versus diseased), among other variables [28].
With such variance across study designs, a clear statement regarding the effectiveness of antioxidant supplements to minimize oxidative stress resulting from exercise is difficult to compose.

The above also appears true for CoQ10 supplementation, with at least two studies involving prophylactic treatment noting attenuation in exercise-induced oxidative stress biomarkers $[29,30]$. Specifically, Tauler and colleagues investigated three months of supplementation involving an antioxidant cocktail, including $100 \mathrm{mg}$ of CoQ10, following a 60-minute soccer match [29]. A noted decrease in plasma oxidative stress was observed with antioxidant supplementation—although details as to which components of the antioxidant cocktail were responsible for the findings are not available. Gül and coworkers noted a partial prevention in exercise-induced lipid peroxidation in a sample of sedentary men following repeated cycle sprints when subjects first supplemented with $100 \mathrm{mg}$ of CoQ10 for eight weeks [30]. In contrast, Braun and coworkers reported no difference in exercise-induced oxidative stress following eight weeks of supplementation with $100 \mathrm{mg}$ per day of CoQ10 in male cyclists [31]. Others note minimal or no difference in exercise-induced oxidative stress following CoQ10 treatment [32]. As with the findings of the present study, it is possible that the inclusion of active subjects who often do not experience a significant increase in oxidative stress in response to acute exercise bouts [6], likely contributed to the findings of no effect. That is, there simply was too small of an increase noted, leaving little room for attenuation. Similar findings have been noted previously with regard to exercise and CoQ10 supplementation in both younger and older individuals [33].

4.4. Physical Performance. With regards to antioxidant supplementation and physical performance, a lack of benefit has been noted in much prior work $[34,35]$. However, CoQ10 
offers more than simply antioxidant effects, as this nutrient is involved in bioenergetic processes [2]. Due to this, the use of CoQ10 as a potential ergogenic aid has been studied for the past several years, and to many individuals, this potential effect appears to be of greater overall importance as compared to the antioxidant effect of this agent. While some studies have noted favorable findings with regards to measures of physical performance $[13,16,36]$, many have not-with few or no differences observed between placebo and CoQ10 supplementation groups $[14,31,33,37]$. In studies in which no effect has been noted, it is possible that the dosage of CoQ10 was too low $[32,37,38]$, in particular considered the relatively poor bioavailability of traditional CoQ10 [1].

In the present study, a sufficient dosage of CoQ10 was provided in order to increase plasma CoQ10 status significantly (Table 3). Despite this elevation, no significant performance benefit was noted for either aerobic or anaerobic exercise tests (Table 2). However, selected individuals appeared to respond to CoQ10 treatment, in particular with regard to the cycle sprint test. Moreover, the relationship between the percentage change in total blood CoQ10 and exercise performance (in particular the cycle sprint total work; $R^{2}=0.6009$ ) was noted to be moderate to strong. These findings provide some indication that higher levels of total blood CoQ10 appear related to higher cycle sprint exercise performance.

Regarding those individuals who appeared to respond to CoQ10 treatment, no discernable pattern of response was noted based on sex or age. For example, of the four subjects who were noted to experience an improvement in treadmill time on the GXT, three were men (aged 30, 40, and 45 years) and one was a woman (aged 44 years). Of the six subjects who were noted to experience an improvement in total work performed during the cycle sprint test, five were men (aged 35, 36, 44, 54, and 57 years) and one was a woman (aged 32 years). Moreover, no noted differences were observed for these "responders" with regard to oxidative stress biomarkers, when compared to those who did not respond to supplementation. These findings are presented in Figure 1 for both total treadmill time (a) and total work during the cycle sprint test (b). This pattern does not shed any light on a given population who may respond best to treatment with CoQ10, but highlights what has been observed for almost all nutritional supplements-some individuals response to treatment and some do not. Additional work with a larger sample of younger and older men and women, in particular of various fitness backgrounds and the use of a variety of physical tests, may more fully elucidate the potential for this nutrient to provide an ergogenic effect.

When considering the above, the results for CoQ10 supplementation as pertaining to both antioxidant effect and physical performance are mixed, with findings mainly influenced by the subjects tested and the type of exercise employed. Our results clearly demonstrate the effect of supplemental CoQ10 on elevating blood levels of CoQ10, while failing to exhibit an antioxidant or ergogenic effect in a sample of exercise-trained subjects. Of course, this statement is specific to the outcome measures included within the present research design. Our results in no way eliminate the possibility for benefit of CoQ10 supplementation on outcomes not measured here.

\section{Conclusion}

The findings presented here indicate that supplementation of CoQ10 in healthy, exercise-trained men and women increases total and reduced blood CoQ10, in addition to CoQ10: Cholesterol ratio. However, no change in resting or exercise-induced measures of oxidative stress is noted, nor is aerobic or anaerobic exercise performance improved following four weeks of supplementation, with the exception of selected individuals. It is possible that more robust effects may be noted within a sample of sedentary and/or older individuals, as well as in those with known disease. In addition, the active subjects in this study had a baseline total CoQ10 value of close to $1.0 \mu \mathrm{g} \cdot \mathrm{mL}^{-1}$, which is higher than the $0.6-0.8 \mu \mathrm{g} \cdot \mathrm{mL}^{-1}$ observed in typical populations, and thus may have contributed to a mitigated response to supplementation. While we used an exercise stressor in the present study in an attempt to increase ROS production and to test the antioxidant potential of CoQ10, other more potent ROS generators such as the ingestion of high-fat feedings may better determine the antioxidant potential of this supplement. Future research is needed to provide answers to the above questions. Lastly, as ubiquinol has demonstrated a distinct effect on gene expression, including activation of genes involved in lipid and lipoprotein metabolism, and countering expression of inflammation (effects not observed with ubiquinone), further research is necessary to relate the benefits of elevated blood values of total and reduced CoQ10 to exercise performance.

\section{Conflict of Interests}

Financial support for this work was provided by Kaneka Nutrients (Pasdena, TX). The investigators and the University of Memphis have no direct or indirect interest in the tested product (Kaneka QH) or in Kaneka Nutrients.

\section{Acknowledgment}

Funding for this work was provided by Kaneka Nutrients (Pasadena, TX).

\section{References}

[1] H. N. Bhagavan and R. K. Chopra, "Plasma coenzyme Q10 response to oral ingestion of coenzyme Q10 formulations," Mitochondrion, vol. 7, pp. S78-S88, 2007.

[2] G. P. Littarru and L. Tiano, "Bioenergetic and antioxidant properties of coenzyme Q10: recent developments," Molecular Biotechnology, vol. 37, no. 1, pp. 31-37, 2007.

[3] S. K. Powers and M. J. Jackson, "Exercise-induced oxidative stress: cellular mechanisms and impact on muscle force production," Physiological Reviews, vol. 88, no. 4, pp. 12431276, 2008. 
[4] S. Palazzetti, M. J. Richard, A. Favier, and I. Margaritis, "Overloaded training increases exercise-induced oxidative stress and damage," Canadian Journal of Applied Physiology, vol. 28, no. 4, pp. 588-604, 2003.

[5] M. Frisard and E. Ravussin, "Energy metabolism and oxidative stress: impact on the metabolic syndrome and the aging process," Endocrine, vol. 29, no. 1, pp. 27-32, 2006.

[6] K. Fisher-Wellman and R. J. Bloomer, "Acute exercise and oxidative stress: a 30 year history," Dynamic Medicine, vol. 8, no. 1, article no. 1, 2009.

[7] L. L. Ji, "Exercise at old age: does it increase or alleviate oxidative stress?" Annals of the New York Academy of Sciences, vol. 928, pp. 236-247, 2001.

[8] M. V. Miles, P. S. Horn, P. H. Tang et al., "Age-related changes in plasma coenzyme Q10 concentrations and redox state in apparently healthy children and adults," Clinica Chimica Acta, vol. 347, no. 1-2, pp. 139-144, 2004.

[9] M. C. Gomez-Cabrera, E. Domenech, M. Romagnoli et al., "Oral administration of vitamin C decreases muscle mitochondrial biogenesis and hampers training-induced adaptations in endurance performance," American Journal of Clinical Nutrition, vol. 87, no. 1, pp. 142-149, 2008.

[10] M. Ristow, K. Zarse, A. Oberbach et al., "Antioxidants prevent health-promoting effects of physical exercise in humans," Proceedings of the National Academy of Sciences of the United States of America, vol. 106, no. 21, pp. 8665-8670, 2009.

[11] P. Niklowitz, A. Sonnenschein, B. Janetzky, W. Andler, and T. Menke, "Enrichment of coenzyme Q10 in plasma and blood cells: defense against oxidative damage," International Journal of Biological Sciences, vol. 3, no. 4, pp. 257-262, 2007.

[12] A. Bonetti, F. Solito, G. Carmosino, A. M. Bargossi, and P. L. Fiorella, "Effect of ubidecarenone oral treatment on aerobic power in middle-aged trained subjects," Journal of Sports Medicine and Physical Fitness, vol. 40, no. 1, pp. 51-57, 2000.

[13] H. Gökbel, I. Gül, M. Belviranli, and N. Okudan, "The effectsof coenzyme Q10 supplementation on performance during repeated bouts of supramaximal exercise in sedentary men," Journal of Strength and Conditioning Research, vol. 24, no. 1, pp. 97-102, 2010.

[14] S. Zhou, Y. Zhang, A. Davie et al., "Muscle and plasma coenzyme Q10 concentration, aerobic power and exercise economy of healthy men in response to four weeks of supplementation," Journal of Sports Medicine and Physical Fitness, vol. 45, no. 3, pp. 337-346, 2005.

[15] S. Beg, S. Javed, and K. Kohli, "Bioavailability enhancement of coenzyme q10: an extensive review of patents," Recent Patents on Drug Delivery and Formulation, vol. 4, no. 3, pp. 245-255, 2010.

[16] K. Mizuno, M. Tanaka, S. Nozaki et al., "Antifatigue effects of coenzyme Q10 during physical fatigue," Nutrition, vol. 24, no. 4, pp. 293-299, 2008.

[17] K. Hosoe, M. Kitano, H. Kishida, H. Kubo, K. Fujii, and M. Kitahara, "Study on safety and bioavailability of ubiquinol (Kaneka QH) after single and 4-week multiple oral administration to healthy volunteers," Regulatory Toxicology and Pharmacology, vol. 47, no. 1, pp. 19-28, 2007.

[18] H. N. Bhagavan and R. K. Chopra, "Coenzyme Q10: absorption, tissue uptake, metabolism and pharmacokinetics," Free Radical Research, vol. 40, no. 5, pp. 445-453, 2006.

[19] H. Ikematsu, K. Nakamura, S. I. Harashima, K. Fujii, and N. Fukutomi, "Safety assessment of coenzyme Q10 (Kaneka Q10) in healthy subjects: a double-blind, randomized, placebocontrolled trial," Regulatory Toxicology and Pharmacology, vol. 44, no. 3, pp. 212-218, 2006.
[20] T. Hidaka, K. Fujii, I. Funahashi, N. Fukutomi, and K. Hosoe, "Safety assessment of coenzyme Q10 (CoQ10)," BioFactors, vol. 32, no. 1-4, pp. 199-208, 2008.

[21] A. M. Jentzsch, H. Bachmann, P. Fürst, and H. K. Biesalski, "Improved analysis of malondialdehyde in human body fluids," Free Radical Biology and Medicine, vol. 20, no. 2, pp. 251-256, 1996.

[22] P. H. Tang, M. V. Miles, A. DeGrauw, A. Hershey, and A. Pesce, "HPLC analysis of reduced and oxidized coenzyme Q10 in human plasma," Clinical Chemistry, vol. 47, no. 2, pp. 256265, 2001.

[23] C. Weber, T. Sejersgard Jakobsen, S. A. Mortensen, G. Paulsen, and G. Holmer, "Antioxidative effect of dietary coenzyme Q10 in human blood plasma," International Journal for Vitamin and Nutrition Research, vol. 64, no. 4, pp. 311-315, 1994.

[24] R. J. Bloomer and K. H. Fisher-Wellman, "Blood oxidative stress biomarkers: influence of sex, exercise training status, and dietary intake," Gender Medicine, vol. 5, no. 3, pp. 218-228, 2008.

[25] L. L. Ji, M. C. Gomez-Cabrera, and J. Vina, "Role of free radicals and antioxidant signaling in skeletal muscle health and pathology," Infectious Disorders. Drug Targets, vol. 9, no. 4, pp. 428-444, 2009.

[26] I. Margaritis and A. S. Rousseau, "Does physical exercise modify antioxidant requirements?" Nutrition Research Reviews, vol. 21, no. 1, pp. 3-12, 2008.

[27] S. L. Williams, N. A. Strobel, L. A. Lexis, and J. S. Coombes, "Antioxidant requirements of endurance athletes: implications for health," Nutrition Reviews, vol. 64, no. 3, pp. 93-108, 2006.

[28] R. J. Bloomer, "Chapter 1 effect of exercise on oxidative stress biomarkers," Advances in Clinical Chemistry, vol. 46, pp. 1-50, 2008.

[29] P. Tauler, M. D. Ferrer, A. Sureda et al., "Supplementation with an antioxidant cocktail containing coenzyme Q prevents plasma oxidative damage induced by soccer," European Journal of Applied Physiology, vol. 104, no. 5, pp. 777-785, 2008.

[30] I. Gül, H. Gökbel, M. Belviranli, N. Okudan, S. Büyükbaş, and K. Basarali, "Oxidative stress and antioxidant defense in plasma after repeated bouts of supramaximal exercise: the effect of coenzyme Q10," Journal of Sports Medicine and Physical Fitness, vol. 51, no. 2, pp. 305-312, 2011.

[31] B. Braun, P. M. Clarkson, P. S. Freedson, and R. L. Kohl, "Effects of coenzyme Q10 supplementation on exercise performance, $\mathrm{VO} 2 \mathrm{max}$, and lipid peroxidation in trained cyclists," International Journal of Sport Nutrition, vol. 1, no. 4, pp. 353$365,1991$.

[32] J. Kaikkonen, L. Kosonen, K. Nyyssönen et al., "Effect of combined coenzyme Q10 and $\mathrm{d}$ - $\alpha$-tocopheryl acetate supplementation on exercise-induced lipid peroxidation and muscular damage: a placebo-controlled double-blind study in marathon runners," Free Radical Research, vol. 29, no. 1, pp. 85-92, 1998.

[33] R. Laaksonen, M. Fogelholm, J. J. Himberg, J. Laakso, and Y. Salorinne, "Ubiquinone supplementation and exercise capacity in trained young and older men," European Journal of Applied Physiology and Occupational Physiology, vol. 72, no. 1-2, pp. 95-100, 1995.

[34] M. Atalay, J. Lappalainen, and C. K. Sen, "Dietary antioxidants for the athlete," Current sports medicine reports, vol. 5, no. 4, pp. 182-186, 2006.

[35] S. Powers, W. B. Nelson, and E. Larson-Meyer, "Antioxidant and vitamin D supplements for athletes: sense or nonsense?" Journal of Sports Sciences, vol. 29, supplement 1, pp. S47-S55, 2011. 
[36] M. Cooke, M. Iosia, T. Buford et al., "Effects of acute and 14day coenzyme Q10 supplementation on exercise performance in both trained and untrained individuals," Journal of the International Society of Sports Nutrition, vol. 5, article no. 8, 2008.

[37] B. Östman, A. Sjödin, K. Michaëlsson, and L. Byberg, "Coenzyme Q10 supplementation and exercise-induced oxidative stress in humans," Nutrition, vol. 28, no. 4, pp. 403-417, 2012.

[38] S. B. Weston, S. Zhou, R. P. Weatherby, and S. J. Robson, "Does exogenous coenzyme Q10 affect aerobic capacity in endurance athletes?" International Journal of Sport Nutrition and Exercise Metabolism, vol. 7, no. 3, pp. 197-206, 1997. 


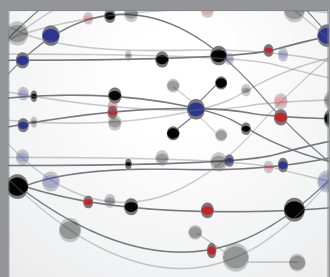

The Scientific World Journal
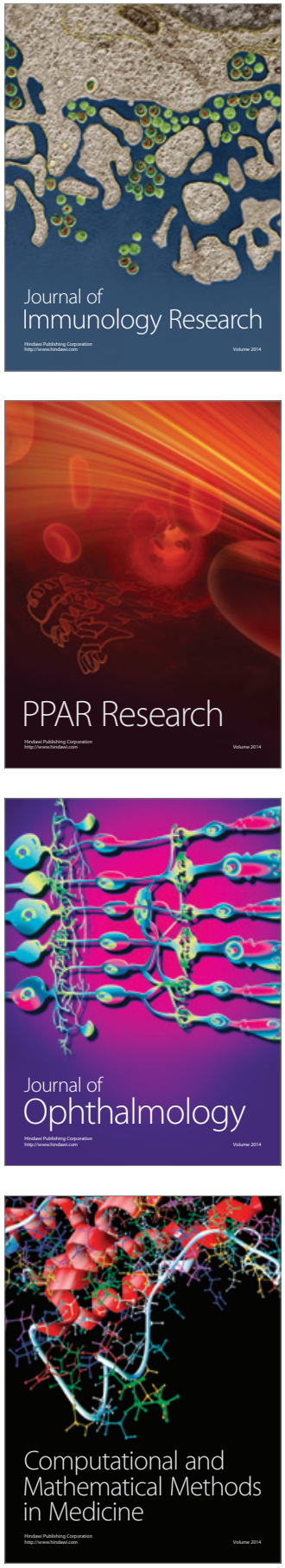

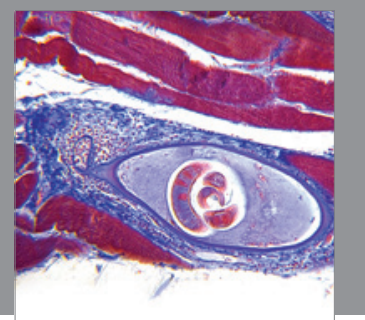

Gastroenterology

Research and Practice
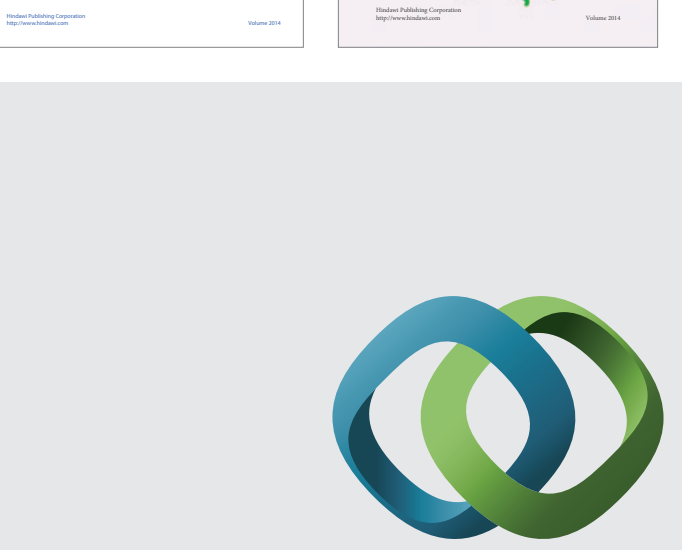

\section{Hindawi}

Submit your manuscripts at

http://www.hindawi.com
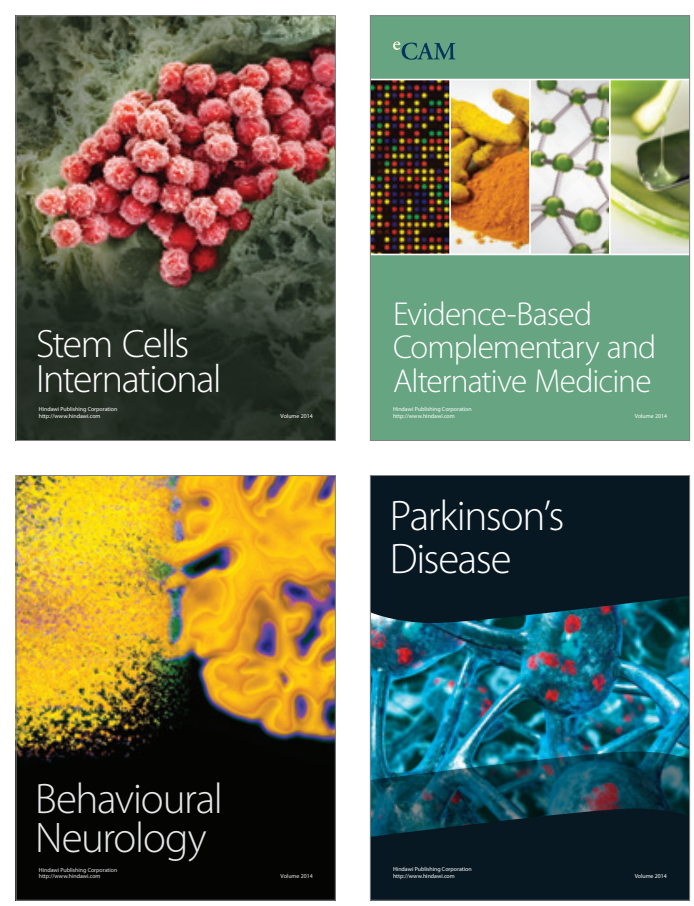

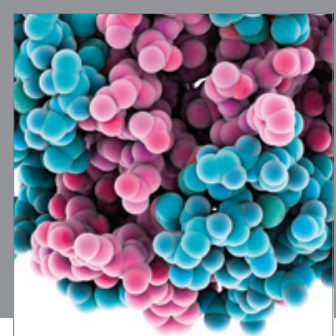

Journal of
Diabetes Research

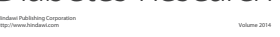

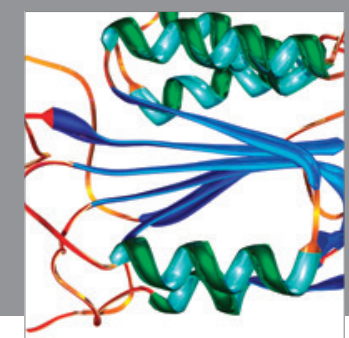

Disease Markers
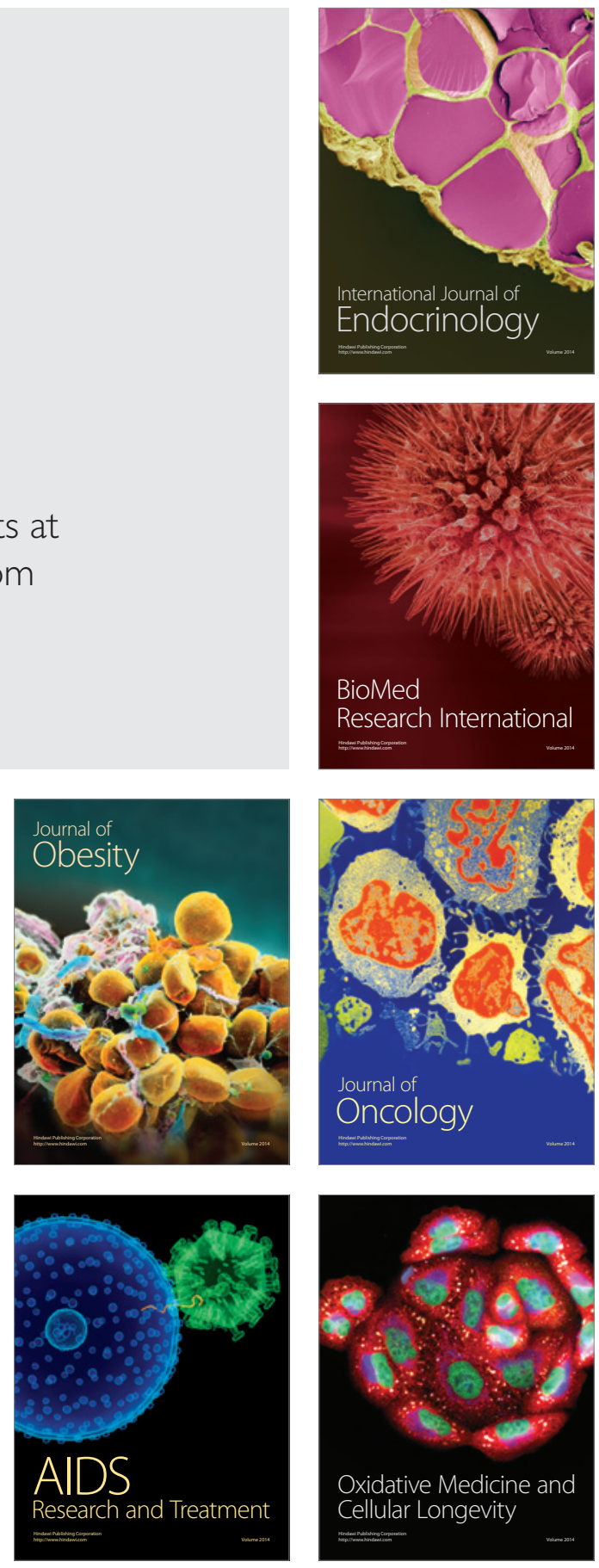